\title{
INTERFERENCE EXPLOITATION PRECODING FOR MULTI-LEVEL MODULATIONS
}

\author{
Ang Li ${ }^{*}$, Christos Masouros ${ }^{\dagger}$, Yonghui Li*, and Branka Vucetic* \\ School of Electrical and Information Engineering, University of Sydney, Sydney, Australia* \\ Department of Electronic and Electrical Engineering, University College London, London, U.K. ${ }^{\dagger}$ \\ Email: \{ang.li2, yonghui.li, branka.vucetic\}@sydney.edu.au*, c.masouros@ucl.ac.uk ${ }^{\dagger}$
}

\begin{abstract}
In this paper, we investigate the interference exploitation precoding for multi-level modulations in the downlink multi-antenna systems. We mathematically derive the optimal precoding structures based on the Karush-Kuhn-Tucker (KKT) conditions. Furthermore, by formulating the dual problem, the precoding problem for multi-level modulations can be transformed into a pre-scaling operation using quadratic programming $(\mathrm{QP})$ optimization. Compared to the original second-order cone programming (SOCP) formulation, this transformation that finally leads to a QP optimization allows a considerable complexity reduction. Simulation results validate our derivations on the optimal precoding structure, and demonstrate significant performance improvements for interference exploitation precoding over traditional precoding methods for multi-level modulations.
\end{abstract}

Index Terms - MIMO, constructive interference, symbol-level precoding, optimization, Lagrangian.

\section{INTRODUCTION}

Precoding has been widely studied as a promising approach to support simultaneous multi-user data transmission in multi-antenna systems. Popular precoding approaches include theoretically capacityachieving dirty paper coding (DPC) [1], linear precoding such as zero-forcing (ZF) and regularized ZF (RZF) [2], and non-linear precoding such as Tomlinson-Harashima precoding (THP) [3] and vector perturbation (VP) precoding [4]. In addition, downlink precoding based on convex optimization has also drawn increasing attention, among which the power minimization [5], [6] and signal-tointerference-plus-noise ratio (SINR) balancing [7]-[9] have been the most popular ones.

For the precoding methods that are described above, the channel state information (CSI) is exploited to design the precoding strategy that eliminates, avoids or limits interference. Nevertheless, the above precoding schemes have ignored the fact that the information of the data symbols can also be exploited for further performance improvements, which is known as the interference exploitation precoding [10]-[14]. It has been shown in [15], [16] that the instantaneous interference can be categorized into constructive interference (CI) and destructive interference. More specifically, CI is defined as the interference that pushes the received signals away from the detection thresholds. In the literature, CI-based precoding has been widely studied in the context of VP in [17], for PSK modulations in [18]-[20], as well as for QAM modulations in [21]. More recently, a sub-optimal closed-form CI precoder has been introduced in [22] for power minimization, and an optimal precoding structure for maxmin CI precoding has been revealed in [23] for PSK modulations. Nevertheless, the extension of the results in [23] to multi-level modulations such as QAM is not trivial, due to the fact that 1) only the outer constellation points can exploit CI, which leads to a different problem formulation, and 2) the phase-rotation CI metric for PSK modulations is not applicable to QAM constellations.

Therefore in this paper, we focus on the interference exploitation precoding for multi-level modulations in the downlink multi-user multiple-input single-output (MU-MISO) system, where we consider QAM as a representative multi-level modulation type. We propose to maximize the $\mathrm{CI}$ effect for the outer constellation points while maintaining the performance for the inner constellations points that cannot exploit CI, which leads to a second-order cone programming (SOCP) formulation. By analyzing the optimization problem with Karush-Kuhn-Tucker (KKT) conditions, we derive the optimal precoding structure as a function of the pre-scaling vector, and obtain an equivalent optimization on the pre-scaling vector. By further formulating the dual of the equivalent optimization, we finally arrive at an equivalent quadratic programming (QP) formulation, which enjoys a reduced computational cost compared to the original SOCP formulation. Simulation results validate our transformations, and demonstrate remarkable performance improvements of CI precoding over traditional precoding approaches for multi-level modulations as well, as opposed to conventional sense that CI precoding is mostly effective for PSK modulations.

Notations: $a, \mathbf{a}$, and $\mathbf{A}$ denote scalar, column vector and matrix, respectively. $(\cdot)^{*},(\cdot)^{T},(\cdot)^{H}$, and $(\cdot)^{-1}$ denote conjugate, transposition, conjugate transposition, and inverse, respectively. $\Re$ and $\Im$ take the real and imaginary part, respectively, and $j$ is the imaginary unit. $\operatorname{diag}(\cdot)$ is the transformation of a column vector into a diagonal matrix, and $\otimes$ is the Kronecker product. $\mathcal{C}^{n \times n}$ and $\mathcal{R}^{n \times n}$ represent an $n \times n$ matrix in the complex and real set, respectively. card $\{\cdot\}$ returns the cardinality of a set. $\mathbf{I}_{K}$ denotes the $K \times K$ identity matrix, and $\mathbf{e}_{i}$ represents the $i$-th column of the identity matrix.

\section{SYSTEM MODEL AND PROBLEM FORMULATION}

We study a downlink MU-MISO system, where the BS with $N_{t}$ transmit antennas communicates with $K$ single-antenna users simultaneously, where $K \leq N_{t}$. The data symbol vector is assumed to be from a normalized QAM constellation, denoted as $\mathbf{s} \in \mathcal{C}^{K \times 1}$. The received signal at user $k$ can then be expressed as

$$
r_{k}=\mathbf{h}_{k}^{T} \mathbf{W} \mathbf{s}+n_{k}, \forall k \in \mathcal{K},
$$

where $\mathcal{K}=\{1,2, \cdots, K\}, \mathbf{h}_{k} \in \mathcal{C}^{N_{t} \times 1}$ denotes the flat-fading Rayleigh channel vector with each entry following the standard complex Gaussian distribution, $\mathbf{W} \in \mathcal{C}^{N_{t} \times K}$ is the precoding matrix, and $n_{k}$ is the additive Gaussian noise at the receiver with zero mean and variance $\sigma^{2}$. As we focus on the analytical precoding structure for CI precoding, perfect CSI is assumed throughout this paper. 
It is known that the conventional phase-rotation CI metric introduced in [18] is not applicable to QAM constellation [21]. Therefore, we employ the symbol-scaling CI metric [17], [24], where we first decompose each constellation point along their detection threshold, expressed as

$$
s_{k}=s_{k}^{\mathcal{A}}+s_{k}^{\mathcal{B}},
$$

where $s_{k}^{\mathcal{A}}$ and $s_{k}^{\mathcal{B}}$ are the bases that are parallel to the detection thresholds. Specifically for QAM, we further obtain

$$
s_{k}^{\mathcal{A}}=\Re\left\{s_{k}\right\}=s_{k}^{\Re}, s_{k}^{\mathcal{B}}=j \cdot \Im\left\{s_{k}\right\}=s_{k}^{\Im} .
$$

For a generic expression of $s_{k}^{\mathcal{A}}$ and $s_{k}^{\mathcal{B}}$ for $\mathcal{M}$-PSK modulations, we refer the interested readers to [25], where we note that the decomposition of QAM is equivalent to that of QPSK. We further decompose each received signal along the detection threshold, given by

$$
\mathbf{h}_{k}^{T} \mathbf{W} \mathbf{s}=\alpha_{k}^{\mathcal{A}} s_{k}^{\mathcal{A}}+\alpha_{k}^{\mathcal{B}} s_{k}^{\mathcal{B}},
$$

where $\alpha_{k}^{\mathcal{A}} \geq 0$ and $\alpha_{k}^{\mathcal{B}} \geq 0$ are two introduced real scalars that represent the effect of interference.

For multi-level modulations such as QAM, CI can only be exploited by the outer constellation points, while all the interference for the inner constellation points is destructive. To be more specific, when QAM modulation is considered, as shown in Fig. 1 below where we employ the 1st quadrant of a 16QAM constellation as an example, CI can only be exploited by the real part of the constellation point ' $\mathrm{B}$ ', the imaginary part of ' $\mathrm{C}$ ', and both real and imaginary part of 'D', respectively. Therefore, we propose to maximize the CI effect for the outer constellation points while maintaining the performance for the inner constellation points, and by further expressing

$$
\boldsymbol{\Omega}_{k}=\left[\alpha_{k}^{\mathcal{A}}, \alpha_{k}^{\mathcal{B}}\right]^{T}, \mathbf{s}_{k}=\left[s_{k}^{\mathcal{A}}, s_{k}^{\mathcal{B}}\right]^{T},
$$

the optimization problem can be constructed as

$$
\begin{array}{ll}
\mathcal{P}_{1}: & \max _{\mathbf{W}, t} t \\
\text { s.t. } & \mathbf{C 1}: \mathbf{h}_{k}^{T} \mathbf{W} \mathbf{s}=\boldsymbol{\Omega}_{k}^{T} \mathbf{s}_{k}, \forall k \in \mathcal{K}, \mathbf{C 2}: t \leq \alpha_{m}^{\mathcal{O}}, \forall \alpha_{m}^{\mathcal{O}} \in \mathcal{O} \\
& \mathbf{C 3}: t=\alpha_{n}^{\mathcal{I}}, \forall \alpha_{n}^{\mathcal{I}} \in \mathcal{I}, \mathbf{C} 4:\|\mathbf{W} \mathbf{s}\|_{2}^{2} \leq p_{0}
\end{array}
$$

where $\mathcal{O}$ consists of the real scalars corresponding to the real or imaginary part of outer constellation points that can exploit CI, while $\mathcal{I}$ consists of the real scalars corresponding to the real or imaginary part of outer constellation points that cannot exploit CI. Accordingly, we obtain

$$
\mathcal{O} \cup \mathcal{I}=\left\{\alpha_{1}^{\mathcal{A}}, \alpha_{1}^{\mathcal{B}}, \alpha_{2}^{\mathcal{A}}, \alpha_{2}^{\mathcal{B}}, \cdots, \alpha_{K}^{\mathcal{A}}, \alpha_{K}^{\mathcal{B}}\right\}
$$



Fig. 1. The symbol-scaling metric for 16QAM
$\mathcal{P}_{1}$ belongs to the SOCP optimization, and can be solved via existing convex optimization tools.

\section{INTERFERENCE EXPLOITATION PRECODING}

Before we present our analysis, we first note that Ws can be viewed as a single vector in $\mathcal{P}_{1}$, and accordingly how the power is distributed among each $\mathbf{w}_{i} s_{i}$ does not affect the optimal solution. Therefore without loss of generality, it is safe to assume that each $\mathbf{w}_{i} s_{i}$ is identical when optimality is achieved, and the power constraint can be equivalently transformed into [23]

$$
\|\mathbf{W} \mathbf{s}\|_{2}^{2} \leq p_{0} \Rightarrow \sum_{i=1}^{K} s_{i}^{*} \mathbf{w}_{i}^{H} \mathbf{w}_{i} s_{i} \leq \frac{p_{0}}{K} .
$$

We further express $\mathcal{P}_{1}$ in a standard minimization form as

$$
\begin{array}{ll}
\mathcal{P}_{2}: & \min _{\mathbf{W}, t}-t \\
\text { s.t. } & \mathbf{C 1}: \mathbf{h}_{k}^{T} \sum_{i=1}^{K} \mathbf{w}_{i} s_{i}-\boldsymbol{\Omega}_{k}^{T} \mathbf{s}_{k}=0, \forall k \in \mathcal{K} \\
& \mathbf{C 2}: t-\alpha_{m}^{\mathcal{O}} \leq 0, \forall \alpha_{m}^{\mathcal{O}} \in \mathcal{O}, \mathbf{C 3}: t-\alpha_{n}^{\mathcal{I}}=0, \forall \alpha_{n}^{\mathcal{I}} \in \mathcal{I} \\
& \mathbf{C 4}: \sum_{i=1}^{K} s_{i}^{*} \mathbf{w}_{i}^{H} \mathbf{w}_{i} s_{i} \leq \frac{p_{0}}{K}
\end{array}
$$

Accordingly, the Lagrangian of $\mathcal{P}_{2}$ is expressed as [26]

$$
\begin{aligned}
& \mathcal{L}\left(\mathbf{w}_{i}, t, \delta_{k}, \kappa_{m}, \tau_{n}, \delta_{0}\right)=-t+\sum_{k=1}^{K} \delta_{k}\left(\mathbf{h}_{k}^{T} \sum_{i=1}^{K} \mathbf{w}_{i} s_{i}-\mathbf{\Omega}_{k}^{T} \mathbf{s}_{k}\right) \\
& +\sum_{m=1}^{\operatorname{card}\{\mathcal{O}\}} \kappa_{m}\left(t-\alpha_{m}^{\mathcal{O}}\right)+\sum_{n=1}^{\operatorname{card}\{\mathcal{I}\}} \tau_{n}\left(t-\alpha_{n}^{\mathcal{I}}\right) \\
& +\delta_{0}\left(\sum_{i=1}^{K} s_{i}^{*} \mathbf{w}_{i}^{H} \mathbf{w}_{i} s_{i}-\frac{p_{0}}{K}\right),
\end{aligned}
$$

where $\delta_{k}, \kappa_{m}, \tau_{n}$ and $\delta_{0}$ are the introduced dual variables, $\delta_{0} \geq 0$, and $\kappa_{m} \geq 0, \forall m \in\{1,2, \cdots, \operatorname{card}\{\mathcal{O}\}\}$. The KKT conditions can be further expressed as

$$
\begin{array}{r}
\frac{\partial \mathcal{L}}{\partial t}=-1+\sum_{m=1}^{\operatorname{card}\{\mathcal{O}\}} \kappa_{m}+\sum_{n=1}^{\operatorname{card}\{\mathcal{I}\}} \tau_{n}=0 \\
\frac{\partial \mathcal{L}}{\partial \mathbf{w}_{i}}=\left(\sum_{k=1}^{K} \delta_{k} \cdot \mathbf{h}_{k}^{T}\right) s_{i}+\delta_{0} s_{i} s_{i}^{*} \cdot \mathbf{w}_{i}^{H}=\mathbf{0}, \forall i \in \mathcal{K} \\
\mathbf{h}_{k}^{T} \sum_{i=1}^{K} \mathbf{w}_{i} s_{i}-\mathbf{\Omega}_{k}^{T} \mathbf{s}_{k}=0, \forall k \in \mathcal{K} \\
\kappa_{m}\left(t-\alpha_{m}^{\mathcal{O}}\right)=0, \forall \alpha_{m}^{\mathcal{O}} \in \mathcal{O} \\
\delta_{0}\left(\sum_{i=1}^{K} s_{i}^{*} \mathbf{w}_{i}^{H} \mathbf{w}_{i} s_{i}-\frac{p_{0}}{K}\right)=0
\end{array}
$$

We first obtain that $\delta_{0} \neq 0$ based on (11b), which further means that $\|\mathbf{W} \mathbf{s}\|_{2}^{2}=p_{0}$ when optimality is achieved. Based on (11b), we can further express $\mathbf{w}_{i}$ as

$$
\mathbf{w}_{i}^{H}=-\frac{s_{i}}{\delta_{0} s_{i} s_{i}^{*}}\left(\sum_{k=1}^{K} \delta_{k} \cdot \mathbf{h}_{k}^{T}\right)=-\frac{1}{s_{i}^{*}}\left(\sum_{k=1}^{K} \frac{\delta_{k}}{\delta_{0}} \cdot \mathbf{h}_{k}^{T}\right) .
$$


By introducing $\vartheta_{k}=-\frac{\delta_{k}^{H}}{\delta_{0}}$, we can further express $\mathbf{w}_{i}$ as

$$
\mathbf{w}_{i}=\left(\sum_{k=1}^{K} \vartheta_{k} \cdot \mathbf{h}_{k}^{*}\right) \frac{1}{s_{i}}, \forall i \in \mathcal{K} .
$$

It is easy to verify that the expression of $\mathbf{w}_{i}$ in (13) is consistent with our premise that each $\mathbf{w}_{i} s_{i}$ is identical. Based on (13), we express the precoding matrix as

$$
\begin{aligned}
\mathbf{W} & =\left[\mathbf{w}_{1}, \mathbf{w}_{2}, \cdots, \mathbf{w}_{K}\right] \\
& =\left[\mathbf{h}_{1}^{*}, \mathbf{h}_{2}^{*}, \cdots, \mathbf{h}_{K}^{*}\right]\left[\vartheta_{1}, \vartheta_{2}, \cdots, \vartheta_{K}\right]^{T}\left[\frac{1}{s_{1}}, \frac{1}{s_{2}}, \cdots, \frac{1}{s_{K}}\right] \\
& =\mathbf{H}^{H} \mathbf{\Upsilon}^{T}
\end{aligned}
$$

where we have introduced

$$
\mathbf{\Upsilon}=\left[\vartheta_{1}, \vartheta_{2}, \cdots, \vartheta_{K}\right]^{T}, \hat{\mathbf{s}}=\left[\frac{1}{s_{1}}, \frac{1}{s_{2}}, \cdots, \frac{1}{s_{K}}\right]^{T} .
$$

We further express (4) in a matrix form, given by

$$
\mathbf{H W}=\left[\boldsymbol{\Omega}_{1}^{T} \mathbf{s}_{1}, \boldsymbol{\Omega}_{2}^{T} \mathbf{s}_{2}, \cdots, \boldsymbol{\Omega}_{K}^{T} \mathbf{s}_{K}\right]^{T}=\mathbf{U} \operatorname{diag}(\boldsymbol{\Omega}) \mathbf{s}_{\mathbf{E}},
$$

where $\mathbf{U} \in \mathcal{R}^{K \times 2 K}=\mathbf{I}_{K} \otimes[1,1]$, and the pre-scaling vector $\boldsymbol{\Omega} \in \mathcal{R}^{2 K \times 1}$ as well as the expanded symbol vector $\mathbf{s}_{\mathbf{E}} \in \mathcal{R}^{2 K \times 1}$ is given by

$$
\begin{gathered}
\boldsymbol{\Omega}=\left[\alpha_{1}^{\mathcal{A}}, \alpha_{1}^{\mathcal{B}}, \cdots, \alpha_{K}^{\mathcal{A}}, \alpha_{K}^{\mathcal{B}}\right]^{T}=\left[\alpha_{1}^{E}, \alpha_{2}^{E}, \cdots, \alpha_{2 K-1}^{E}, \alpha_{2 K}^{E}\right]^{T}, \\
\mathbf{s}_{\mathbf{E}}=\left[s_{1}^{\mathcal{A}}, s_{1}^{\mathcal{B}}, \cdots, s_{K}^{\mathcal{A}}, s_{K}^{\mathcal{B}}\right]^{T}=\left[s_{1}^{E}, s_{2}^{E}, \cdots, s_{2 K-1}^{E}, s_{2 K}^{E}\right]^{T} .
\end{gathered}
$$

Then, by substituting the precoding matrix $\mathbf{W}$ in (14) into (17), we obtain the expression of $\Upsilon$ as

$$
\begin{aligned}
& \mathbf{H H}^{H} \mathbf{\Upsilon} \hat{\mathbf{s}}^{T} \mathbf{s}=\mathbf{U} \operatorname{diag}(\boldsymbol{\Omega}) \mathbf{s}_{\mathbf{E}} \\
\Rightarrow & \boldsymbol{\Upsilon}=\frac{1}{K} \cdot\left(\mathbf{H H}^{H}\right)^{-1} \mathbf{U} \operatorname{diag}(\boldsymbol{\Omega}) \mathbf{s}_{\mathbf{E}},
\end{aligned}
$$

which further leads to the expression of the precoding matrix $\mathbf{W}$ as a function of the pre-scaling vector $\Omega$ as

$$
\mathbf{W}=\frac{1}{K} \cdot \mathbf{H}^{H}\left(\mathbf{H} \mathbf{H}^{H}\right)^{-1} \mathbf{U} \operatorname{diag}(\boldsymbol{\Omega}) \mathbf{s}_{\mathbf{E}} \hat{\mathbf{S}}^{T} .
$$

Subsequently, we substitute $\mathbf{W}$ obtained in (19) into the power constraint in $\mathcal{P}_{1}$, and we obtain

$$
\begin{gathered}
\|\mathbf{W} \mathbf{s}\|_{2}^{2}=p_{0} \Rightarrow \mathbf{s}_{\mathbf{E}}^{H} \operatorname{diag}(\boldsymbol{\Omega}) \mathbf{U}^{H}\left(\mathbf{H H}^{H}\right)^{-1} \mathbf{U} \operatorname{diag}(\boldsymbol{\Omega}) \mathbf{s}_{\mathbf{E}}=p_{0} \\
\Rightarrow \boldsymbol{\Omega}^{T} \underbrace{\operatorname{diag}\left(\mathbf{s}_{\mathbf{E}}^{H}\right) \mathbf{U}^{H}\left(\mathbf{H H}^{H}\right)^{-1} \mathbf{U} \operatorname{diag}\left(\mathbf{s}_{\mathbf{E}}\right)}_{\mathbf{T}} \boldsymbol{\Omega}=p_{0},
\end{gathered}
$$

where we note $\mathbf{s}^{H} \hat{\mathbf{s}}^{*}=\hat{\mathbf{s}}^{T} \mathbf{s}=K$. With $\mathbf{T}$ being symmetric and positive semi-definite and $\Omega$ being real, (20) is equivalent to

$$
\boldsymbol{\Omega}^{T} \mathbf{T} \boldsymbol{\Omega}=p_{0} \Rightarrow \boldsymbol{\Omega}^{T} \Re\{\mathbf{T}\} \boldsymbol{\Omega}=p_{0} \Rightarrow \boldsymbol{\Omega}^{T} \mathbf{V} \boldsymbol{\Omega}=p_{0},
$$

based on which we can construct an optimization on $\Omega$, given by

$$
\begin{array}{ll}
\mathcal{P}_{3}: & \min _{\boldsymbol{\Omega}, t}-t \\
\text { s.t. } & \mathbf{C 1}: \boldsymbol{\Omega}^{T} \mathbf{V} \boldsymbol{\Omega}-p_{0}=0, \mathbf{C 2}: t-\alpha_{m}^{\mathcal{O}} \leq 0, \forall \alpha_{m}^{\mathcal{O}} \in \mathcal{O} \\
& \mathbf{C 3}: t-\alpha_{n}^{\mathcal{I}}=0, \forall \alpha_{n}^{\mathcal{I}} \in \mathcal{I}
\end{array}
$$

The optimal precoding matrix can then be obtained by substituting the optimal $\Omega$ from $\mathcal{P}_{3}$ into (19).

We further analyze $\mathcal{P}_{3}$ and derive the optimal precoding structure as a function of the dual variables of $\mathcal{P}_{3}$. The Lagrangian of $\mathcal{P}_{3}$ is formulated as

$$
\begin{aligned}
& \mathcal{L}\left(\boldsymbol{\Omega}, t, \tilde{\delta}_{0}, \mu_{m}, \nu_{n}\right)=-t+\tilde{\delta}_{0}\left(\boldsymbol{\Omega}^{T} \mathbf{V} \boldsymbol{\Omega}-p_{0}\right) \\
& +\sum_{m=1}^{\operatorname{card}\{\mathcal{O}\}} \mu_{m}\left(t-\alpha_{m}^{\mathcal{O}}\right)+\sum_{n=1}^{\operatorname{card}\{\mathcal{I}\}} \nu_{n}\left(t-\alpha_{n}^{\mathcal{I}}\right)
\end{aligned}
$$

where $\mu_{m} \geq 0, \forall m \in\{1,2, \cdots, \operatorname{card}\{\mathcal{O}\}\}$. To derive a closedform expression, we re-order the columns and rows of the matrices and vectors included in (23). To be more specific, we first re-order the expanded symbol vector $\mathbf{S}_{\mathbf{E}}$ into

$$
\mathbf{S}_{\mathbf{E}} \Rightarrow \tilde{\mathbf{s}}_{\mathbf{E}}=\left[\tilde{\mathbf{s}}_{\mathcal{O}}^{T}, \tilde{\mathbf{s}}_{\mathcal{I}}^{T}\right]^{T}
$$

where the entries in $\tilde{\mathbf{s}}_{\mathcal{O}} \in \mathcal{R}^{\operatorname{card}\{\mathcal{O}\} \times 1}$ correspond to the real and imaginary part of the data symbols that can be scaled, while $\tilde{\mathbf{s}}_{\mathcal{I}} \in$ $\mathcal{R}^{\operatorname{card}\{\mathcal{I}\} \times 1}$ corresponds to the symbol vector that cannot exploit CI. $\tilde{\mathbf{s}}_{\mathcal{O}}$ and $\tilde{\mathbf{s}}_{\mathcal{I}}$ are given by

$$
\tilde{\mathbf{s}}_{\mathcal{O}}=\left[\tilde{s}_{1}, \cdots, \tilde{s}_{\operatorname{card}\{\mathcal{O}\}}\right]^{T}, \tilde{\mathbf{s}}_{\mathcal{I}}=\left[\tilde{s}_{\operatorname{card}\{\mathcal{O}\}+1}, \cdots, \tilde{s}_{2 K}\right]^{T},
$$

The pre-scaling vector $\Omega$ is accordingly re-ordered into

$$
\boldsymbol{\Omega} \Rightarrow \tilde{\boldsymbol{\Omega}}=\left[\tilde{\mathbf{\Omega}}_{\mathcal{O}}^{T}, \tilde{\mathbf{\Omega}}_{\mathcal{I}}^{T}\right]^{T}
$$

where $\tilde{\boldsymbol{\Omega}}_{\mathcal{O}} \in \mathcal{R}^{\operatorname{card}\{\mathcal{O}\} \times 1}$ and $\tilde{\boldsymbol{\Omega}}_{\mathcal{I}} \in \mathcal{R}^{\operatorname{card}\{\mathcal{I}\} \times 1}$ are given by

$$
\tilde{\mathbf{\Omega}}_{\mathcal{O}}=\left[\tilde{\alpha}_{1}, \cdots, \tilde{\alpha}_{\operatorname{card}\{\mathcal{O}\}}\right]^{T}, \tilde{\mathbf{\Omega}}_{\mathcal{I}}=\left[\tilde{\alpha}_{c a r d}\{\mathcal{O}\}+1, \cdots, \tilde{\alpha}_{2 K}\right]^{T} .
$$

We further introduce a Locater function that returns the index of $\tilde{s}_{m}$ in the original expanded symbol vector $\mathbf{s}_{\mathbf{E}}$, given by

$$
\mathbf{L}\left(\tilde{s}_{m}\right)=k, \text { if } \tilde{s}_{m}=s_{k}^{E} .
$$

We can then express $\tilde{\mathbf{S}}_{\mathbf{E}}$ and $\tilde{\boldsymbol{\Omega}}$ as a linear transformation of $\mathbf{S}_{\mathbf{E}}$ and $\Omega$, given by

$$
\tilde{\mathbf{s}}_{\mathbf{E}}=\mathbf{F} \mathbf{s}_{\mathbf{E}}, \quad \tilde{\boldsymbol{\Omega}}=\mathbf{F} \boldsymbol{\Omega},
$$

where the transformation matrix $\mathbf{F} \in \mathcal{R}^{2 K \times 2 K}$ is given by

$$
\mathbf{F}=\left[\mathbf{e}_{\mathbf{L}\left(\tilde{s}_{1}\right)}, \mathbf{e}_{\mathbf{L}\left(\tilde{s}_{2}\right)}, \cdots, \mathbf{e}_{\mathbf{L}\left(\tilde{s}_{2 K}\right)}\right]^{T},
$$

where we note that $\mathbf{F}$ is invertible. Similarly, the coefficient matrix $\mathbf{V}$ is accordingly re-ordered into

$$
\tilde{\mathbf{V}}=\mathbf{F V F}^{T},
$$

where the multiplication of $\mathbf{F}$ at the left side and $\mathbf{F}^{T}$ at the right side correspond to the row and column reordering, respectively. Based on the above transformations, the Lagrangian in (23) can be simplified into

$$
\mathcal{L}\left(\tilde{\mathbf{\Omega}}, t, \tilde{\delta}_{0}, \tilde{\mathbf{u}}\right)=\left(\mathbf{1}^{T} \tilde{\mathbf{u}}-1\right) t+\tilde{\delta}_{0} \cdot \tilde{\mathbf{\Omega}}^{T} \tilde{\mathbf{V}} \tilde{\mathbf{\Omega}}-\tilde{\mathbf{u}}^{T} \tilde{\mathbf{\Omega}}_{-} \tilde{\delta}_{0} p_{0}
$$

where $\mathbf{1}=[1,1, \cdots, 1]^{T} \in \mathcal{R}^{2 K \times 1}$ and the dual vector $\tilde{\mathbf{u}} \in$ $\mathcal{R}^{2 K \times 1}$ is given by

$$
\tilde{\mathbf{u}}=\left[\tilde{\mu}_{1}, \tilde{\mu}_{2}, \cdots, \tilde{\mu}_{c a r d\{\mathcal{O}\}}, \tilde{\nu}_{1}, \tilde{\nu}_{2}, \cdots, \tilde{\nu}_{\operatorname{card}\{\mathcal{I}\}}\right]^{T} .
$$


The KKT conditions for $\mathcal{P}_{3}$ can then expressed as

$$
\begin{array}{r}
\frac{\partial \mathcal{L}}{\partial t}=\mathbf{1}^{T} \tilde{\mathbf{u}}-1=0 \\
\frac{\partial \mathcal{L}}{\partial \tilde{\mathbf{\Omega}}}=\tilde{\delta}_{0} \cdot\left(\tilde{\mathbf{V}}+\tilde{\mathbf{V}}^{T}\right) \tilde{\mathbf{\Omega}}-\tilde{\mathbf{u}}=\mathbf{0} \\
\tilde{\mathbf{\Omega}^{T}} \tilde{\mathbf{V}} \tilde{\mathbf{\Omega}}-p_{0}=0 \\
\tilde{\mu}_{m}\left(t-\tilde{\alpha}_{m}\right)=0, \forall m \in\{1,2, \cdots, \operatorname{card}\{\mathcal{O}\}\} \\
t-\tilde{\alpha}_{n}=0, \forall n \in\{\operatorname{card}\{\mathcal{I}\}+1, \cdots, 2 K\}
\end{array}
$$

Based on (34b), we obtain the expression of $\tilde{\Omega}$ as

$$
\tilde{\mathbf{\Omega}}=\frac{1}{2 \tilde{\delta}_{0}} \cdot \tilde{\mathbf{V}}^{-1} \tilde{\mathbf{u}}
$$

By substituting the expression of $\tilde{\Omega}$ into the power constraint, we further obtain the expression of $\tilde{\delta}_{0}$ as

$$
\begin{aligned}
& \left(\frac{1}{2 \tilde{\delta}_{0}} \cdot \tilde{\mathbf{V}}^{-1} \tilde{\mathbf{u}}\right)^{T} \tilde{\mathbf{V}}^{-1}\left(\frac{1}{2 \tilde{\delta}_{0}} \cdot \tilde{\mathbf{V}}^{-1} \tilde{\mathbf{u}}\right)=p_{0} \\
\Rightarrow & \frac{1}{4 \tilde{\delta}_{0}^{2}} \cdot \tilde{\mathbf{u}}^{T} \tilde{\mathbf{V}}^{-1} \tilde{\mathbf{V}} \tilde{\mathbf{V}}^{-1} \tilde{\mathbf{u}}=p_{0} \\
\Rightarrow & \tilde{\delta}_{0}=\sqrt{\frac{\tilde{\mathbf{u}}^{T} \tilde{\mathbf{V}}-1}{4 p_{0}}} .
\end{aligned}
$$

For $\mathcal{P}_{3}$, it is easy to observe that the Slater's condition is satisfied [26], and therefore $\mathcal{P}_{3}$ can be optimally solved by solving its dual problem, which is constructed as

$$
\mathcal{P}_{4}: \mathcal{D}=\max _{\tilde{\mathbf{u}}, \tilde{\delta}_{0}} \min _{\tilde{\mathbf{\Omega}}, t} \mathcal{L}\left(\tilde{\mathbf{u}}, \tilde{\delta}_{0}, \tilde{\mathbf{\Omega}}, t\right) .
$$

The inner minimization of $\mathcal{P}_{4}$ is achieved with (34a) and (35), which leads to

$$
\begin{aligned}
\mathcal{D} & =\max _{\tilde{\mathbf{u}}, \tilde{\delta}_{0}} \tilde{\delta}_{0} \cdot \tilde{\Omega}^{T} \tilde{\mathbf{V}} \tilde{\boldsymbol{\Omega}}+\tilde{\mathbf{u}}^{T} \tilde{\boldsymbol{\Omega}}-\tilde{\delta}_{0} p_{0} \\
& =\max _{\tilde{\mathbf{u}}, \tilde{\delta}_{0}} \frac{\tilde{\delta}_{0}}{4 \tilde{\delta}_{0}^{2}} \cdot \tilde{\mathbf{u}}^{T} \tilde{\mathbf{V}}^{-1} \tilde{\mathbf{V}} \tilde{\mathbf{V}}^{-1} \tilde{\mathbf{u}}-\frac{1}{2 \tilde{\delta}_{0}} \tilde{\mathbf{u}}^{T} \tilde{\mathbf{V}}^{-1} \tilde{\mathbf{u}}-\tilde{\delta}_{0} p_{0} \\
& =\max _{\tilde{\mathbf{u}}}-\frac{\tilde{\mathbf{u}}^{T} \tilde{\mathbf{V}}^{-1} \tilde{\mathbf{u}}}{4 \sqrt{\frac{\tilde{\mathbf{u}}^{T} \tilde{\mathbf{V}}^{-1} \tilde{\mathbf{u}}}{4 p_{0}}}}-\sqrt{\frac{\tilde{\mathbf{u}}^{T} \tilde{\mathbf{V}}^{-1} \tilde{\mathbf{u}}}{4 p_{0}}} \cdot p_{0} \\
& =\max _{\tilde{\mathbf{u}}}-\sqrt{p_{0} \cdot \tilde{\mathbf{u}}^{T} \tilde{\mathbf{V}}-1} \tilde{\mathbf{u}} \\
& =\min _{\tilde{\mathbf{u}}} \tilde{\mathbf{u}}^{T} \tilde{\mathbf{V}}^{-1} \tilde{\mathbf{u}},
\end{aligned}
$$

and accordingly the dual problem is equivalent to

$$
\begin{array}{ll}
\mathcal{P}_{5}: & \min _{\tilde{\mathbf{u}}} \tilde{\mathbf{u}}^{T} \tilde{\mathbf{V}}^{-1} \tilde{\mathbf{u}} \\
\text { s.t. } & \mathbf{C 1}: \mathbf{1}^{T} \tilde{\mathbf{u}}-1=0 \\
& \mathbf{C 2}: \tilde{\mu}_{m} \geq 0, \forall m \in\{1,2, \cdots, \operatorname{card}\{\mathcal{O}\}\}
\end{array}
$$

which is a QP optimization and can be much more efficiently solved than the original SOCP formulation $\mathcal{P}_{1}$. Moreover, based on (35) and (36), we obtain a closed-form expression of the optimal precoding matrix as a function of the dual vector $\tilde{\mathbf{u}}$, given by

$$
\begin{aligned}
& \mathbf{W}= \\
& \frac{1}{K} \cdot \mathbf{H}^{H}\left(\mathbf{H H}^{H}\right)^{-1} \mathbf{U} \operatorname{diag}\left(\sqrt{\frac{p_{0}}{\mathbf{u}_{\mathbf{1}}^{T} \tilde{\mathbf{V}}^{-1} \mathbf{u}_{\mathbf{1}}}} \mathbf{F}^{-1} \tilde{\mathbf{V}}^{-1} \mathbf{u}_{\mathbf{1}}\right) \mathbf{S}_{\mathbf{E}} \hat{\mathbf{S}}^{T},
\end{aligned}
$$

with $\mathbf{F}$ given in (30).

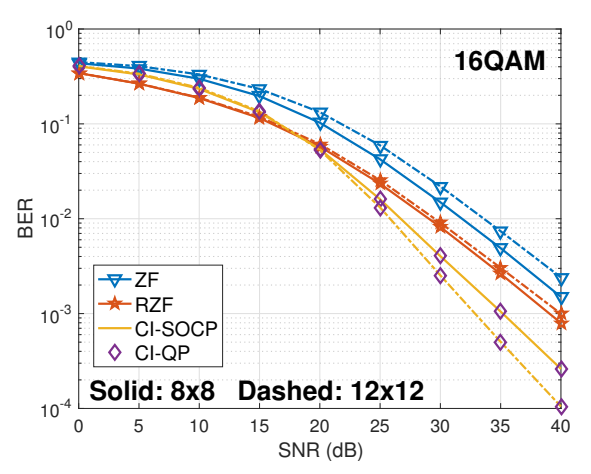

Fig. 2. BER v.s. SNR, $K=N_{t}=8$ and $K=N_{t}=12$, 16QAM

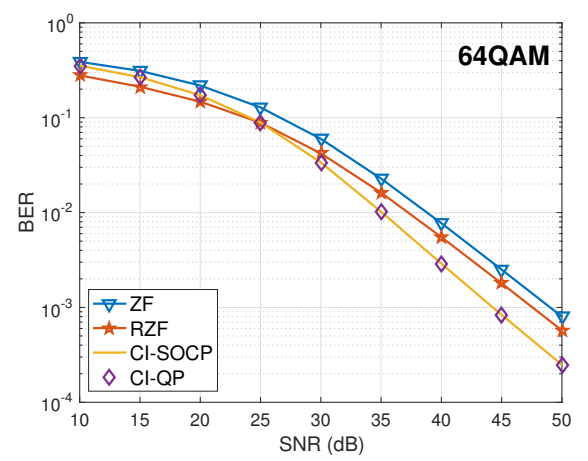

Fig. 3. BER v.s. SNR, $K=N_{t}=12$, 64QAM

\section{NUMERICAL RESULTS}

We compare our QP-based CI precoding with traditional SOCPbased CI precoding as well as other linear ZF and RZF precoding schemes [2] based on Monte Carlo simulations. We assume $p_{0}=1$, and the transmit SNR per antenna as $\rho=1 / \sigma^{2}$.

In Fig. 2 and Fig. 3, we present the bit error rate (BER) results of CI precoding for 16QAM and 64QAM, respectively. In both figures, we observe that the QP-based CI precoding well matches the original SOCP-based CI precoding, which validates the effectiveness of our derivations. Moreover, for both modulations, considerable performance improvements are observed for CI-based precoding over traditional ZF and RZF precoding schemes in the medium-to-high SNR regime. An important observation is that, as opposed to conventional sense that CI precoding is mostly effective for PSK modulations, we show that the performance gains can be as large as $10 \mathrm{~dB}$ for 16QAM and 5dB for 64QAM.

\section{CONCLUSIONS}

In this paper, we study the interference exploitation precoding for multi-level modulations. By analyzing the optimization problem with KKT conditions and by formulating the dual problem, it is revealed that $\mathrm{CI}$ precoding is equivalent to a $\mathrm{QP}$ formulation, and we derive the optimal closed-form precoding matrix as a function of the dual vector of the QP optimization. Our derivations have been validated by the numerical results, which also demonstrate significant performance improvements for interference exploitation precoding over conventional linear precoding schemes. 


\section{REFERENCES}

[1] M. Costa, "Writing on Dirty Paper," IEEE Trans. Inf. Theory, vol. IT-29, no. 3, pp. 439-441, May 1983.

[2] C. B. Peel, B. M. Hochwald, and A. L. Swindlehurst, "A Vector-Perturbation Technique for Near-Capacity Multiantenna Multiuser Communication-part I: Channel Inversion and Regularization," IEEE Trans. Commun., vol. 53, no. 1, pp. 195-202, Jan. 2005.

[3] L. Sun and M. Lei, "Quantized CSI-based TomlinsonHarashima Precoding in Multiuser MIMO Systems," IEEE Trans. Wireless Commun., vol. 12, no. 3, pp. 1118-1126, Mar. 2013.

[4] B. M. Hochwald, C. B. Peel, and A. L. Swindlehurst, "A Vector-Perturbation Technique for Near-Capacity Multiantenna Multiuser Communication-part II: Perturbation," IEEE Trans. Commun., vol. 53, no. 3, pp. 537-544, Mar. 2005.

[5] A. Wiesel, Y. C. Eldar, and S. Shamai (Shitz), "Linear Precoding via Conic Optimization for Fixed MIMO Receivers," IEEE Trans. Sig. Process., vol. 54, no. 1, pp. 161-176, Jan. 2006.

[6] M Bengtsson and B. Ottersten, "Optimal and Suboptimal Transmit Beamforming," Handbook of Antennas in Wireless Communications, Jan. 2001.

[7] M. F. Hanif, L.-N. Tran, A. Tolli, and M. Juntti, "Computationally Efficient Robust Beamforming for SINR Balancing in Multicell Downlink with Applications to Large Antenna Array Systems," IEEE Trans. Commun., vol. 62, no. 6, pp. 19081920, June 2014.

[8] F. Wang, X. Wang, and Y. Zhu, "Transmit Beamforming for Multiuser Downlink with Per-Antenna Power Constraints," in 2014 IEEE International Conference on Communications (ICC), Sydney, NSW, 2014, pp. 4692-4697.

[9] M. Schubert and H. Boche, "Solution of the Multiuser Downlink Beamforming Problem with Individual SINR Constraints," IEEE Trans. Veh. Tech., vol. 53, no. 1, pp. 18-28, Jan. 2004.

[10] C. Masouros, T. Ratnarajah, M. Sellathurai, C. B. Papadias, and A. K. Shukla, "Known Interference in the Cellular Downlink: A Performance Limiting Factor or a Source of Green Signal Power?," IEEE Commun. Mag., vol. 51, no. 10, pp. 162171, Oct. 2013.

[11] G. Zheng, I. Krikidis, C. Masouros, S. Timotheou, D. A. Toumpakaris, and Z. Ding, "Rethinking the Role of Interference in Wireless Networks," IEEE Commun. Mag., vol. 52, no. 11, pp. 152-158, Nov. 2014

[12] A. Li, C. Masouros, Y. Li, B. Vucetic, and A. L. Swindlehurst, "Interference Exploitation Precoding for Multi-Level Modulations: Closed-Form Solutions," arXiv preprint, available online: https://arxiv.org/abs/1811.03289, 2018.

[13] A. Li, C. Masouros, Y. Li, and B. Vucetic, "Multiplexing More Streams in the MU-MISO Downlink by Interference Exploitation Precoding," arXiv preprint, available online: https://arxiv.org/abs/1901.03844, 2019.

[14] M. Alodeh, D. Spano, A. Kalantari, C. Tsinos, D. Christopoulos, S. Chatzinotas, and B. Ottersten, "Symbol-Level and Multicast Precoding for Multiuser Multiantenna Downlink: A Survey, Classification and Challenges," IEEE Commun. Surveys \& Tut., vol. 20, no. 3, pp. 1733-1757, thirdquarter 2018.
[15] C. Masouros and E. Alsusa, "Dynamic Linear Precoding for the Exploitation of Known Interference in MIMO Broadcast Systems," IEEE Trans. Wireless Commun., vol. 8, no. 3, pp. 1396-1401, Mar. 2009.

[16] C. Masouros, "Correlation Rotation Linear Precoding for MIMO Broadcast Communicaitons," IEEE Trans. Sig. Process., vol. 59, no. 1, pp. 252-262, Jan. 2011.

[17] C. Masouros, M. Sellathurai, and T. Ratnarajah, "Vector Perturbation based on Symbol Scaling for Limited Feedback MISO Downlinks," IEEE Trans. Sig. Process., vol. 62, no. 3, pp. 562-571, Feb. 2014.

[18] C. Masouros and G. Zheng, "Exploiting Known Interference as Green Signal Power for Downlink Beamforming Optimization," IEEE Trans. Sig. Process., vol. 63, no. 14, pp. 36283640, July 2015.

[19] M. Alodeh, S. Chatzinotas, and B. Ottersten, "Constructive Multiuser Interference in Symbol Level Precoding for the MISO Downlink Channel," IEEE Trans. Sig. Process., vol. 63, no. 9, pp. 2239-2252, May 2015.

[20] M. Alodeh, S. Chatzinotas, and B. Ottersten, "Energy-Efficient Symbol-Level Precoding in Multiuser MISO based on Relaxed Detection Region," IEEE Trans. Wireless Commun., vol. 15, no. 5, pp. 3755-3767, May 2016.

[21] M. Alodeh, S. Chatzinotas, and B. Ottersten, "Symbol-Level Multiuser MISO Precoding for Multi-Level Adaptive Modulation," IEEE Trans. Wireless Commun., vol. 16, no. 8, pp. 5511-5524, Aug. 2017.

[22] A. Haqiqatnejad, F. Kayhan, and B. Ottersten, "Power Minimizer Symbol-Level Precoding: A Closed-Form Suboptimal Solution,” IEEE Sig. Process. Lett., vol. 25, no. 11, pp. 17301734, Nov. 2018.

[23] A. Li and C. Masouros, "Interference Exploitation Precoding Made Practical: Optimal Closed-Form Solutions for PSK Modulations," IEEE Trans. Wireless Commun., vol. 17, no. 11, pp. 7661-7676, Nov. 2018.

[24] A. Li and C. Masouros, "Exploiting Constructive Mutual Coupling in P2P MIMO by Analog-Digital Phase Alignment," IEEE Trans. Wireless Commun., vol. 16, no. 3, pp. 1948-1962, Mar. 2017.

[25] A. Li, C. Masouros, F. Liu, and A. L. Swindlehurst, "Massive MIMO 1-Bit DAC Transmission: A Low-Complexity Symbol Scaling Approach," IEEE Trans. Wireless Commun., vol. 17, no. 11, pp. 7559-7575, Nov. 2018.

[26] L. Vandenberghe and S. Boyd, Convex Optimization, Cambridge University Press, 2004. 